\title{
A degradation model for simultaneous brightness and sharpness enhancement of low-light image
}

\author{
Pengliang $\mathrm{Li}^{1}$, Junli Liang ${ }^{1 *}$, Miaohua Zhang ${ }^{2}$ \\ ${ }^{1}$ School of Electronics and Information, Northwestern Polytechnical University, China. \\ ${ }^{2}$ Institute for Integrated and Intelligent Systems, Griffith University, Australia.
}

\begin{abstract}
Although a large number of methods have been proposed for low-light image enhancement, there still remain challenges for these methods to simultaneously achieve excellent sharpness/resolution, high calculation efficiency as well as visual pleasure requirements. In this communication, we propose a new low-light image enhancement method based on the degradation model to overcome this dilemma. Specifically, we regard the low-light image enhancement as a special inverse problem of image degradation, and then the task of low-light enhancement is logically embedded in the iterative back-projection (IBP) framework. Meanwhile, an adaptive gamma correction is utilized to adaptively adjust the brightness, and then the IBP framework is transferred to the logarithmic domain instead of the spatial domain for further acceleration. Besides, a simple and effective pre-processing strategy is proposed to pre-enhance the low-light input while making the enhanced image clarify (or visual pleasure). Extensive experimental results on public databases and seven state-of-the-art benchmarks consistently demonstrate the effectiveness and efficiency of the proposed method both visually and quantitatively.
\end{abstract}

Keywords: Low-light image enhancement; Adaptive gamma correction; Logarithmic domain acceleration; Iterative back-projection.

\section{Introduction}

In the low-light environment, the captured images often suffers from low visibility and low sharpness/resolution [1] which are not beneficial to visual pleasure and subsequent computer vision tasks (e.g., target classification, detection, and tracking, etc.). To overcome this issue, the sharpness and brightness of the low-light image need to be enhanced. Currently, low-light image enhancement methods can be roughly divided into two categories: optimization modelbased methods and deep-learning-based methods. For optimization model-based methods, one representative group of methods achieved the image enhancement by modifying their histograms, which is simple and effective [2]. For example, Lee et al. 3] proposed a layered difference representation of 2D histograms to enhance the contrast, and Huang et al. 4 combined an adaptive gamma correction and probability distribution to modify histograms for enhancing the contrast of the low-light image. A fusion-based method for enhancing the weakly illuminated images was proposed in [ $[$ in which the derived inputs obtained from the estimated illumination and corresponding weights 
are multiplied to enhance the weakly illuminated component while avoiding artifacts. In [6], an accurate camera response model and the estimated exposure ratio map were combined to adjust each pixel to its desired exposure. In [7, a structure and texture aware Retinex model was employed to enhance the low-light image. Besides the above mentioned methods, more and more image enhancement methods were developed from different point of view, such as the absorption light scattering method [8] and the enhancement model based on RGB and near-infrared with different exposure times 9 , etc.

In recent years, deep-learning-based methods have been successfully applied to low-light image enhancement task. For example, Wei et al. [10] made use of the low/normal-light image pairs and developed a deep Retinex-Net in which a Decom-Net used for decomposition and an Enhance-Net was utilized for illumination adjustment. Then Zhang et al. [11] added a restoration network to the network designed by Wei et al. [10] to achieve the purpose of enhancement. Wang et al. [12] employed the generative adversarial network following the Retinex decomposition network to enhance the low-light image.

While massive research works have been proposed for low-light image enhancement in the past years, the exploration on the sharpness/resolution degradation of the low-light image has rarely been considered so far. To make up for this gap, in this communication, we propose a novel degradation model to enhance the sharpness and brightness of the low-light image simultaneously. The specific innovations/technical contributions of this work are summarized as follows.

- A new degradation model is developed between the normal-light sharpness image and the low-light image, and then an iterative back-projection (IBP) framework is proposed to solve the resulted degradation model efficiently. Besides, a logarithmic domain transformation method is proposed to accelerate the optimization process.

- We propose an adaptive gamma correction method with the illumination, which can better improve the brightness in the low-light area compared to $\gamma=2.2$ (a widely adopted empirical value) while slightly suppressing overexposure in the high-light area.

- A simple and effective pre-processing step is proposed to pre-enhance the low-light input while keeping visual pleasure.

The rest of this communication is organized as follows. Problem modelling and formulation are presented in Section 2. The proposed model and the optimization process are given in Section 3. Experimental results are presented in Section 4. Finally, Conclusions are drawn in Section 5. 


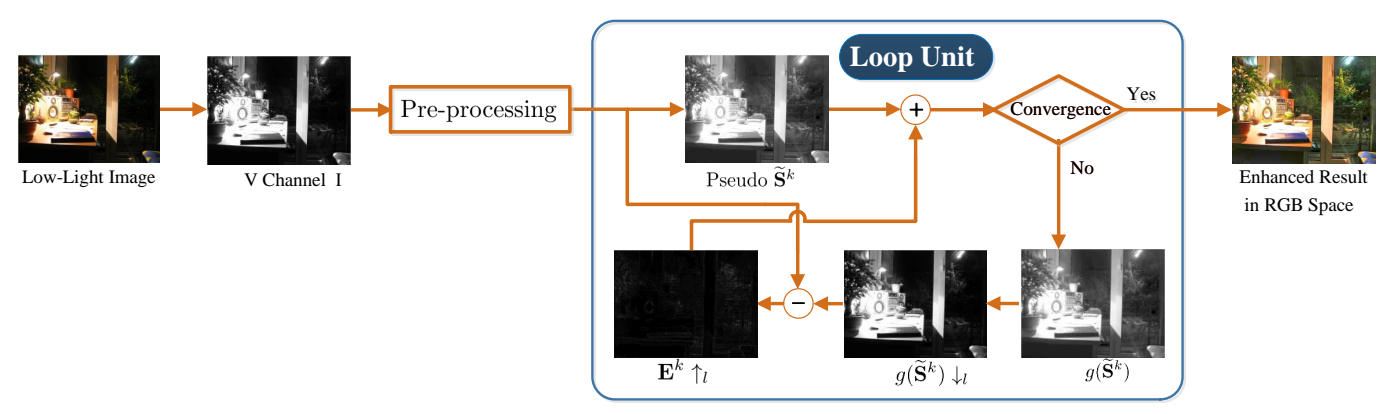

Figure 1: Overview of the proposed method for low-light image enhancement.

\section{Modelling and formulation}

In this section, we describe the degradation process of a low-light image as a normal-light sharpness image through blurring and brightness darkening. Given a normal-light sharpness image $\mathbf{S}$ and a low-light image $\mathbf{I}$, the relationship between them is expressed as

$$
\mathbf{I}=g(\mathbf{S}) \downarrow_{l},
$$

where $g(\cdot)$ represents the blurring degradation function, and $\downarrow_{l}$ is the brightness darkening operator.

Since solving $\mathbf{S}$ from $\mathbf{I}$ in Equation (1) involves an inverse problem, it requires additional regularization terms to constrain the energy lost to approach a desired solution and thus needs huge computational resources [13]. To reduce the calculational burden while ensuring the desired enhancement performance, we utilize the iterative back-projection (IBP) framework [14] to minimize the reconstruction error of the proposed degradation model (i.e., Equation (1)), in which the desired solution is quickly obtained through iterations without additional regularization. Following this framework, we first introduce a pseudo $\widetilde{\mathbf{S}}^{k}(k=0)$ as the initialization of the desired image to produce a pseudo $\widetilde{\mathbf{I}}^{k}$ $(k=0)$ as

$$
\widetilde{\mathbf{I}}^{k}=g\left(\widetilde{\mathbf{S}}^{k}\right) \downarrow_{l},
$$

where $\widetilde{\mathbf{S}}^{k}$ is a normal-light image but lacks the high-frequency details, and because of this property, the degraded pseudo $\widetilde{\mathbf{I}}^{k}$ is smoother than the original low-light image $\mathbf{I}$. Then, the reconstruction error $\mathbf{E}^{k}$ can be generalized by the difference between $\mathbf{I}$ and $\widetilde{\mathbf{I}}^{k}$ as

$$
\mathbf{E}^{k}=\mathbf{I}-\widetilde{\mathbf{I}}^{k}
$$

With the reconstruction error $\mathbf{E}^{k}$, the enhanced image $\widetilde{\mathbf{S}}^{k+1}$ is obtained by accumulating reconstruction error $\mathbf{E}^{k}$ into the pseudo $\widetilde{\mathbf{S}}^{k}$ as

$$
\widetilde{\mathbf{S}}^{k+1}=\widetilde{\mathbf{S}}^{k}+f\left(\mathbf{E}^{k} \uparrow_{l}\right),
$$

where $\uparrow_{l}$ represents the brightness improving operator, and $f(\cdot)$ denotes the inverse smoothing function. A desired $\widetilde{\mathbf{S}}^{k+1}$ can be obtained by repeating Equations (2) to (4) until the algorithm converges. An overview of the proposed method is shown in Figure 1. In the proposed method, we set $f(x)=x$, which is proved to be feasible by experimental 
results in Section 4.

\section{Proposed method}

In this section, we develop the detailed model for the problem proposed in Section 2 to simultaneously achieve excellent sharpness/resolution, high calculation efficiency, and visual pleasure for the enhanced low-light image. Firstly, we introduce a new pre-processing step, and then reformulate the model stated in Section 2 with adaptive gamma correction and logarithmic transformation. Besides, the degenerate functions $g(\cdot)$ and $\downarrow_{l}$ are re-marked for the sake of the readability, for example, the $g(\cdot)$ operator is replaced with the Gaussian filter $g($ i.e., $g(x)=x * g$, where $*$ is the convolution operation), $\downarrow_{l}$ or $\uparrow_{l}$ operator are replaced with the gamma $\gamma$ correction (i.e., $x \downarrow=x^{\frac{1}{\gamma}}, x \uparrow=x^{\gamma}$ where $x>0$ and $\gamma<1$ ), and the pseudo $\widetilde{\mathbf{S}}^{0}$ is replaced with $\mathbf{I} \uparrow_{l}$.

\subsection{Pre-processing}

Since we only get a high-sharpness/resolution version of the gamma correction by solving Equation (1), it is not visually pleasing (or visually clear) enough as shown in Figure. 2 (c). To make up for this shortcoming, we propose a simple yet effective pre-processing step in which the input is set as

$$
\overline{\mathbf{I}}=\mathbf{I} \odot \mathbf{I} \oslash G(\mathbf{I}),
$$

where $\oslash, \odot$, and $G(\cdot)$ represent the element-wise division operator, element-wise multiplication operator, and the piece-wise smooth function, respectively. In our experiments, we use guided image filtering [15] instead of $G(\cdot)$. The squared input divided by the piece-wise smoothed input can produce the pre-enhanced high-frequency details and meanwhile with great clarity in $\overline{\mathbf{I}}$, which can be visually proved by comparing Figure 2 (b) with Figure 2 (d) (especially, the area corresponding to the yellow arrow in Figure 2(a)). Thus, the final result, as shown in Figure 2 (e), is of great visual pleasure by comparing with Figure 2 (c).

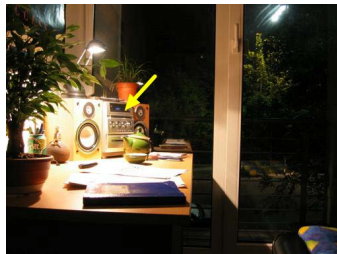

(a) Low-light image

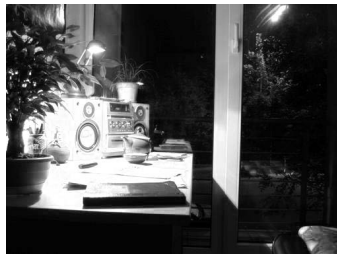

(b) Input I (V channel)

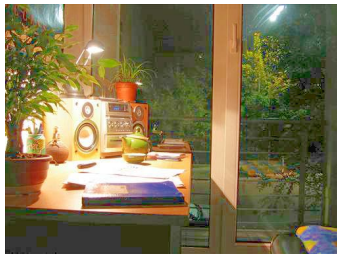

(c) Enhanced result via $\mathbf{I}$

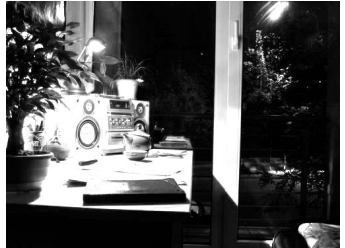

(d) $\overline{\mathbf{I}}$

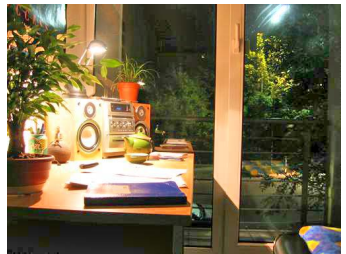

(e) Enhanced result via $\overline{\mathbf{I}}$

Figure 2: An example to illustrate the necessity of pre-processing.

\subsection{Adaptive gamma correction}

As one of the fast low-light image enhancement algorithms, gamma correction improves the pixel intensity in the low-light area via the exponential function. However, gamma correction tends to result in insufficient brightness 
improvement in the low-light area and overexposure in the high-light area as shown in Figure 3 (d). To alleviate this dilemma, we propose an adaptive gamma correction method which acts on the image illumination and achieves better enhancement performance compared with the model with $\gamma=2.2$ (a widely adopted empirical value) in the low-light area and also suppress the overexposure in the high-light area to a large extend, which can be seen from Figures $3(\mathrm{~d}-\mathrm{e})$

Specifically, we set the proposed adaptive gamma correction function as $y=x^{a(1+x)}$, where $x(0 \leq x \leq 1)$ is the input and $y$ is its corrected counterpart with adaptive gamma $\gamma_{a}\left(\gamma_{a}=a(1+x)\right)$. The reasons for choosing parameter $a$ are: 1) compared with $a=0.3$ (or 0.4 ), we set $a$ to 0.35 to better improve the brightness in the low-light area while appropriately suppressing overexposure in the high-light area as shown in Figure $3(\mathrm{a}) ; 2)$ the model with $\gamma_{a}=0.35$ (i.e., $x=0$ ) can properly increase the brightness of low-light area compared to that with $\frac{1}{\gamma}=\frac{1}{2.2}=0.4545$, which can be seen in the yellow square area of Figures $3(\mathrm{~d})$ and $(\mathrm{e}) ; 3) \gamma_{a}$ approaches to 0.7 when $x$ is close to 1 , the proposed model with this parameter can suppress overexposure in the high-light area compared to that with $\frac{1}{\gamma}=0.4545$ as shown in the cyan square area in Figures $3(\mathrm{~d})$ and (e). For comparison, the result of adaptive gamma correction with weighting distribution (AGCWD) method [4] is also represented in Figure 3 (c).

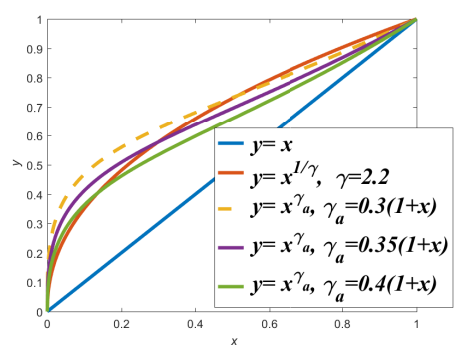

(a)

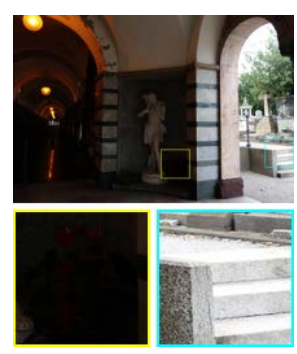

(b)

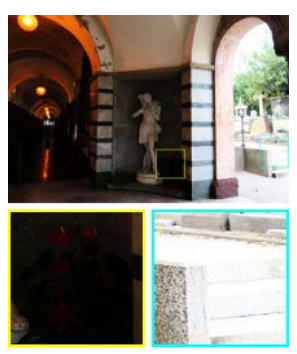

(c)

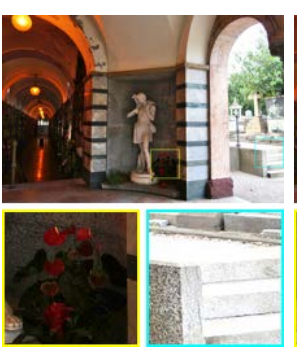

(d)

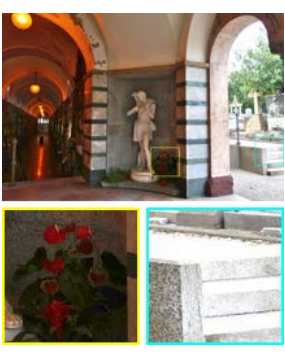

(e)

Figure 3: An example to illustrate the advantages of the proposed adaptive gamma correction. (a) visualization of different gamma curves, (b) the input low-light image, (c) the enhanced result of AGCWD [4], (d) the enhanced result of the proposed method with $\frac{1}{\gamma}=\frac{1}{2.2}$, (e) the enhanced result of the proposed method with $\gamma_{a}(a=0.35)$.

\subsection{Logarithmic domain acceleration}

Since the computational complexity of logarithmic linear function $O(n \log a)$ is lower than that of exponential function $O\left(a^{n}\right)$ [16, we transform the proposed model in Section 1 from spatial domain to the logarithmic domain, in which two exponential operations are converted into two logarithmic linear operations in each iteration, thus the calculation efficiency of the proposed method can be much improved. In logarithmic domain, the pre-processing, brightness improving operator, and darkening operator are respectively defined by

$$
\left\{\begin{aligned}
\log (\overline{\mathbf{I}})=2 \log (\mathbf{I})-\log (G(\mathbf{I})) & \rightleftharpoons \overline{\mathbf{I}}=\mathbf{I} \odot \mathbf{I} \oslash G(\mathbf{I}), \\
\gamma_{a} \odot \log (\overline{\mathbf{I}}) & \rightleftharpoons \overline{\mathbf{I}}^{\gamma_{a}}, \\
\log (\overline{\mathbf{I}}) \oslash \gamma_{a} & \rightleftharpoons \overline{\mathbf{I}}^{\frac{1}{\gamma_{a}}}
\end{aligned}\right.
$$


where $\rightleftharpoons$ denotes the domain transformation.

According to above transformations, we combine the adaptive gamma correction and logarithmic domain acceleration to develop a new low-light image enhancement method called AGCLDA which is summarized in Algorithm 1.

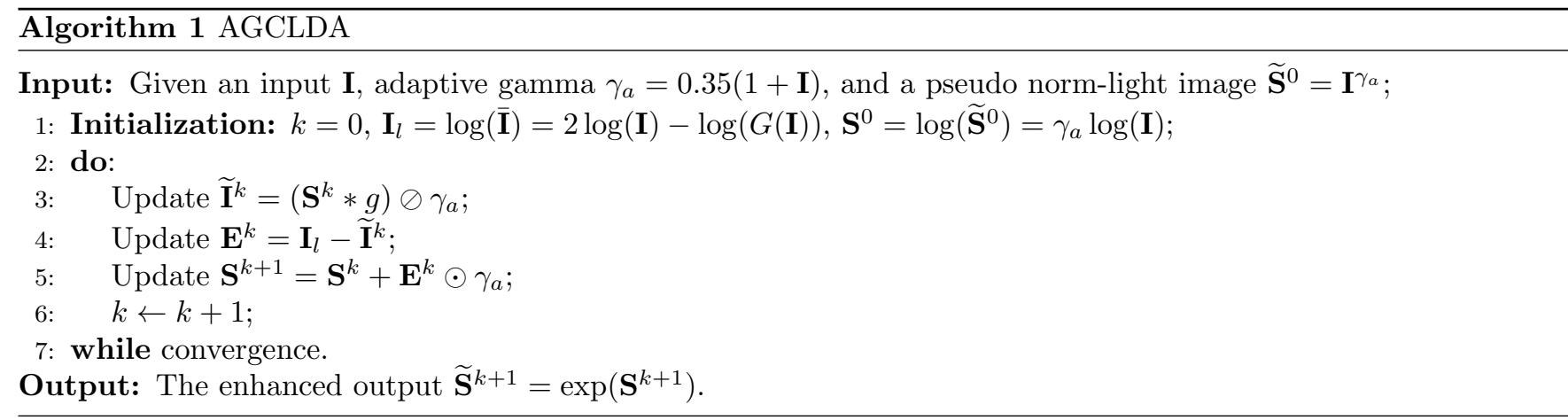

\subsection{Convergence analysis}

We here give the proof of convergence for the proposed AGCLDA method as follows:

$$
\begin{aligned}
\mathbf{E}^{k+1} & =\mathbf{I}_{l}-\tilde{\mathbf{I}}^{k+1} \\
& =\mathbf{I}_{l}-\left(1 \oslash \gamma_{a}\right) \odot\left(\mathbf{S}^{k+1} * g\right) \\
& =\mathbf{I}_{l}-\left(1 \oslash \gamma_{a}\right) \odot\left(\left(\mathbf{S}^{k}+\gamma_{a} \odot\left(\mathbf{I}_{l}-\left(1 \oslash \gamma_{a}\right) \odot\left(\mathbf{S}^{k} * g\right)\right)\right) * g\right) \\
& =\mathbf{I}_{l}-\left(1 \oslash \gamma_{a}\right) \odot\left(\mathbf{S}^{k} * g\right)-\left(1 \oslash \gamma_{a}\right) \odot\left(\left(\gamma_{a} \odot\left(\mathbf{I}_{l}-\left(1 \oslash \gamma_{a}\right) \odot\left(\mathbf{S}^{k} * g\right)\right)\right) * g\right) \\
& =\left(1 \oslash \gamma_{a}\right) \odot\left(\gamma_{a} \odot\left(\mathbf{I}_{l}-\left(1 \oslash \gamma_{a}\right) \odot\left(\mathbf{S}^{k} * g\right)\right)\right)-\left(1 \oslash \gamma_{a}\right) \odot\left(\left(\gamma_{a} \odot\left(\mathbf{I}_{l}-\left(1 \oslash \gamma_{a}\right) \odot\left(\mathbf{S}^{k} * g\right)\right)\right) * g\right) \\
& =\left(1 \oslash \gamma_{a}\right) \odot\left(\gamma_{a} \odot \mathbf{E}^{k}\right)-\left(1 \oslash \gamma_{a}\right) \odot\left(\left(\gamma_{a} \odot \mathbf{E}^{k}\right) * g\right) .
\end{aligned}
$$

Then both sides of Equation (7) are multiplied by $\gamma_{a}$

$$
\gamma_{a} \odot \mathbf{E}^{k+1}=\gamma_{a} \odot \mathbf{E}^{k}-\left(\gamma_{a} \odot \mathbf{E}^{k}\right) * g=\left(\gamma_{a} \odot \mathbf{E}^{k}\right) *(\delta-g) .
$$

According to Youn's inequality [17, 18, we have

$$
\left\|\gamma_{a} \odot \mathbf{E}^{k+1}\right\|_{1}=\left\|\left(\gamma_{a} \odot \mathbf{E}^{k}\right) *(\delta-g)\right\|_{1} \leq\left\|\gamma_{a} \odot \mathbf{E}^{k}\right\|_{1} \times\|\delta-g\|_{1}
$$

Since $\gamma_{a}$ is a constant matrix, the value of $\left\|\mathbf{E}^{k+1}\right\|_{1}\left(\right.$ or $\left.\left\|\gamma_{a} \odot \mathbf{E}^{k+1}\right\|_{1}\right)$ will close to 0 when both $\|\delta-g\|_{1}<1$ and $k \rightarrow \infty$ hold. Thus, we obtain an expected $\mathbf{S}^{k+1}$ satisfying $\mathbf{I}_{l}=\left(\mathbf{S}^{k+1} * g\right) \oslash \gamma_{a}$ when the proposed method converges.

\section{Experimental results}

In this section, we carry out experiments to test the performance of the proposed adaptive gamma correction and logarithmic domain acceleration (AGCLDA) method and compare it with seven state-of-the-art methods, including model-based methods: adaptive gamma correction method (AGCWD) [4], layered difference representation of 2D 
histograms (LDR) [3], camera response model (CRM) 6], fusion-based method (FBM) [5], a structure and texture aware Retinex method (STAR) [7, and two deep-learning-based methods: deep Retinex decomposition (RetinexNet) [10] and kindling the darkness (KinD) [11. We collect 16 low-light images for testing from [19, 12, 20, 21] as shown in Figure 4. For the proposed AGCLDA method, the V channel of each low-light image is enhanced in the HSV color space, and then the enhanced image is obtained by converting it from HSV to RGB color space. All the experiments are conducted on a PC with Intel i5-4590 CPU 3.30GHz and 16GB memory.

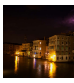

(a)

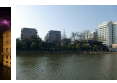

(b)

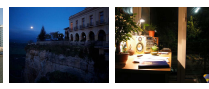

(c) (d)

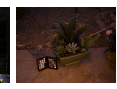

(e)

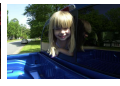

(f)

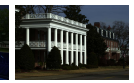

(g)

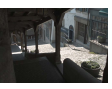

(h)

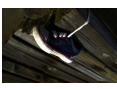

(i)

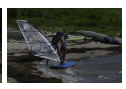

(j)

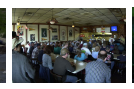

(k)

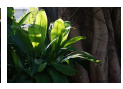

(1)

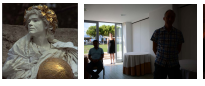

(m) (n)

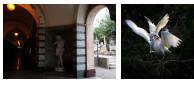

(o)

(p)

\subsection{Evaluation metrics}

In our experiments, we select two noreference image quality assessment methods for quantitative evaluation. One is the natural image quality evaluator (NIQE) for reflecting the naturalness [22], and another is the autoregressivebased image sharpness metric (ARISM) for estimating the degree of sharpness/blurriness [23]. A small value produced by NIQE means better image quality of the naturalness, while a larger value of ARISM denotes better sharpness (or lower blurriness). To reflect the comprehensive performance (CP), we define a new quality evaluator as

$$
\mathrm{CP}=\frac{\mathrm{ARISMC}}{\mathrm{NIQE}}
$$

according to the above analysis, we know that a larger value of $\mathrm{CP}$ indicates a better comprehensive performance, i.e., both the naturalness and sharpness are excellent.

\subsection{Performance of low-light image enhancement}

For the proposed method, we fix the size of Gaussian filter $g$ to $5 \times 5$. Figure 5 shows the effect of variance $\sigma$ of $g$ on the sharpness of the enhanced image. If a high-sharpness output is needed, one can choose a larger $\sigma$.

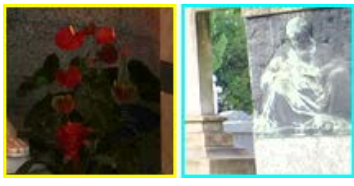

(a) $\sigma=0.3$

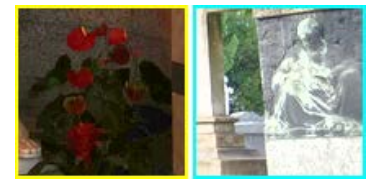

(b) $\sigma=0.4$

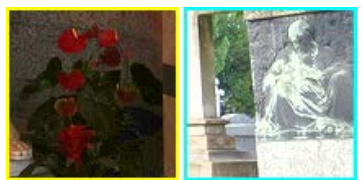

(c) $\sigma=0.5$

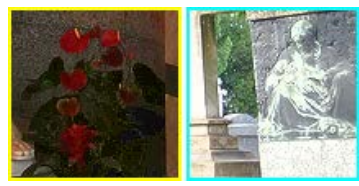

(d) $\sigma=0.6$

Figure 5: A visualization to illustrate the influence of $\sigma$ on the image sharpness.

In Table 1, we list the influence of different $\sigma$ (satisfying the convergence condition $\|\delta-g\|_{1}<1$ ) on the NIQE, ARISMC, and CP for the proposed AGCLDA method. By comparing with the state-of-the-art methods in Table 2 . we set $\sigma=0.3$, which makes CP rank the 1st (indicated with the bold). To analyse the convergence of the proposed method, we use the maximum absolute error (MAE, and $\mathrm{MAE}=\max \left(\left|\mathbf{E}\left(\mathbf{S}^{k+1}\right)-\mathbf{E}\left(\mathbf{S}^{k}\right)\right|\right)$ to measure the error of 


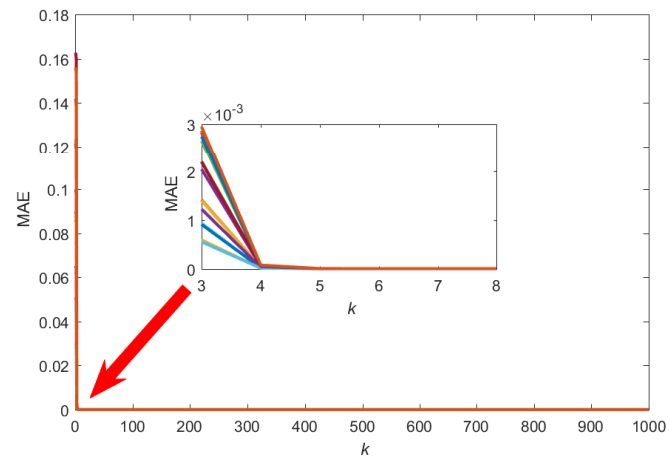

Figure 6: The convergence curves of 16 low-light testing images with $\sigma=0.3$.

Table 1: The influence of different $\sigma$ on the NIQE, ARISMC, and CP for the proposed AGCLDA method

\begin{tabular}{l|cccccccccccc}
\hline \multicolumn{1}{c|}{$\sigma$} & 0.1 & 0.15 & 0.2 & 0.25 & 0.3 & 0.35 & 0.4 & 0.45 & 0.5 & 0.55 & 0.6 \\
\hline NIQE & 2.6937 & 2.6937 & 2.6936 & 2.6960 & 2.7000 & 2.7458 & 2.8740 & 3.2076 & 3.8514 & 4.7994 & 5.8323 \\
\hline ARISMC & 2.8030 & 2.8030 & 2.8030 & 2.8036 & 2.8147 & 2.8417 & 2.8999 & 2.9800 & 3.0343 & 3.0950 & 3.2111 \\
\hline CP & 1.0405 & 1.0405 & 1.0406 & 1.0399 & $\mathbf{1 . 0 4 2 4}$ & 1.0349 & 1.0090 & 1.0368 & 0.7878 & 0.6448 & 0.5505 \\
\hline
\end{tabular}

each iteration. In Figure 6, we plot MAEs of each iteration for 16 low-light testing images in Figure 4 . Since the value of all MAEs is less than 0.001 with $k=4$, we set the maximum iteration number to 4 .

Table 2: Comparison of NIQE, ARISMC, CP, and average run time (ART) using different methods

\begin{tabular}{l|clllclll}
\hline Method & AGCWD [4] & LDR [3] & CRM [6] & FBM [5] & RetinexNet [10] & KinD [11] & STAR [7] & AGCLDA \\
\hline NIQE & 2.8203 & 2.7932 & $\underline{2.7003}$ & 2.7867 & 3.6028 & 2.9515 & 2.7241 & $\mathbf{2 . 7 0 0 0}$ \\
\hline ARISMC & 2.7268 & 2.7274 & 2.7772 & 2.7576 & $\mathbf{2 . 8 9 2 5}$ & 2.5658 & 2.7285 & $\underline{2.8147}$ \\
\hline CP & 0.9668 & 0.9764 & $\underline{1.0284}$ & 0.9895 & 0.8028 & 0.8693 & 1.0016 & $\mathbf{1 . 0 4 2 4}$ \\
\hline ART $(\mathrm{s})$ & $\mathbf{0 . 0 5 1 1}$ & 1.8238 & 0.2755 & 0.2915 & - & - & 4.0210 & $\underline{0.1456}$ \\
\hline
\end{tabular}

To quantitatively analyse the superiority of the proposed AGCLDA method, we list the evaluation indicators of NIQE, ARISMC, CP, and average running time (ART) in Table 2, and compare them with that of seven state-of-theart benchmark methods. From these results we can draw the follow conclusions: 1) although the ART of AGCWD [4] method is the fastest, the CP of the enhanced result is not satisfactory; 2) the RetinexNet 10 method achieves the best ARISMC in the case of losing the naturalness, while the CP of the RetinexNet method ranks the lowest; 3) the CRM method achieves superior comprehensive performance and ranks the 2nd, 4) the comprehensive performance of the remaining comparison methods is moderate, 5) compared with the seven state-of-the-art benchmark methods, the proposed AGCLDA method achieves the best CP and superior computational efficiency, and ranks the 1st and 2nd (indicated with the underlined) in terms of NIQE and ARISM respectively. Besides, the comprehensive performance of the proposed method can be further proved by comparing local areas of the enhanced images with seven state-of-the-art benchmark methods as shown in Figure 7 and Figure 8 


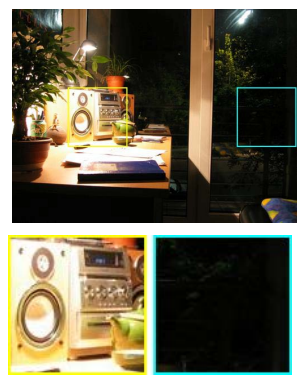

(a) Input

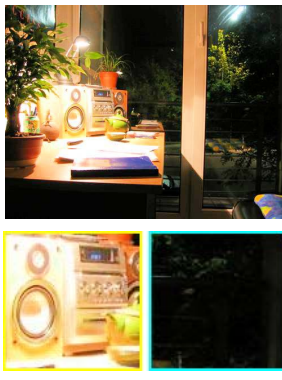

(b) AGCWD [4]

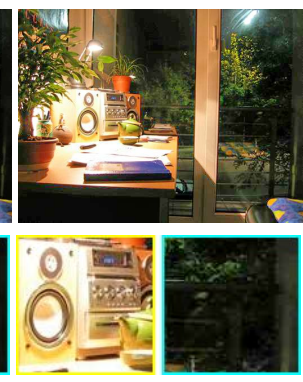

(c) FBM [5]

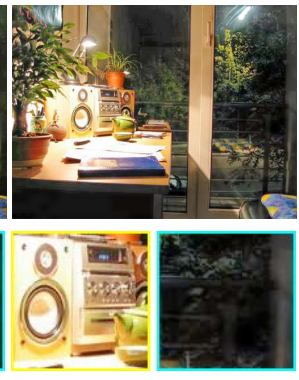

(d) KinD [1]

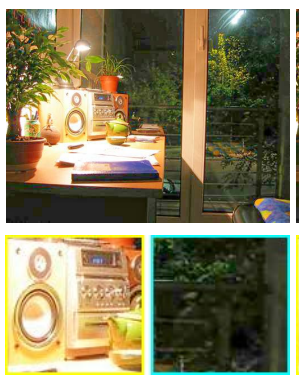

(e) STAR[7]

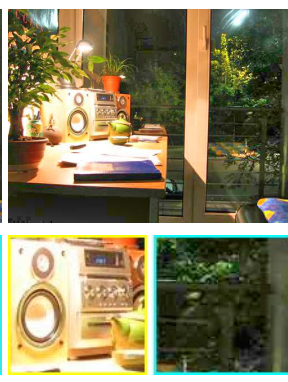

(f) AGCLDA

Figure 7: The enhanced images are displayed for a more detailed comparison of each method.

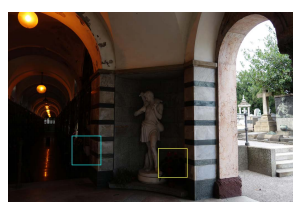

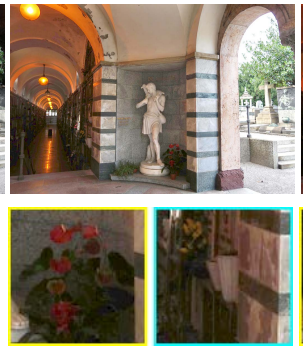

(b) $\mathrm{CRM} 6$

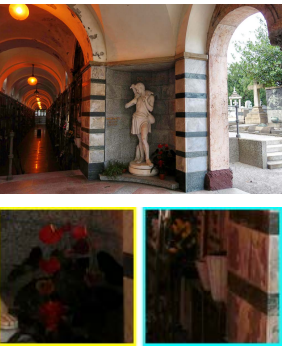

(c) FBM $[5$

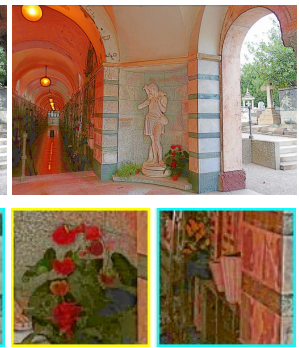

(d) RetinexNet [0]

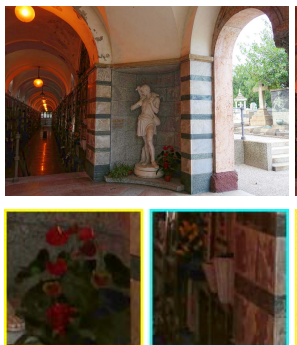

(e) STAR 7

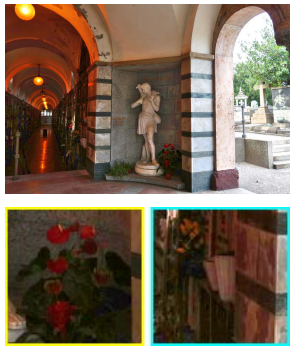

(f) AGCLDA

Figure 8: The enhanced images are displayed for a more detailed comparison of each method.

\subsection{Computational complexity analysis}

To further evaluate the effectiveness of the proposed method, we analyse the computational complexity for each method. For fair comparison, here we only give detailed complexities for model-based methods since the neuralnetwork-based methods have different training strategy. Let the input size of an image be $m \times n$, the main complexities of the AGCWD [4] and LDR [3] methods, which were proposed from the perspective of histogram, are $O\left(256^{0.5}+256^{c}\right)$ and $O\left(\log (m n)+\sum_{i}^{255}(256-i) i\right)$, respectively, where $c$ is linearly related to probability density function. For the CRM [6] method, the main computational complexity is $O\left(m n+(m n)^{-0.3293}+e^{m n}\right)$. The main computational complexity of FBM [5] is $O\left(e^{m n}+S m n\right)$, where $S$ represents the number of convolution kernel. For the STAR [7] method, the main computational complexity is $O\left(K m n+L\left((m n)^{0.5}+(m n)^{1.5}\right)\right)$, where $K$ and $L$ indicate the numbers of internal and external iterations, respectively. In Algorithm 1, the proposed AGCLDA method requires to update $\widetilde{\mathbf{I}}^{k}$ and $\mathbf{S}^{k}$ whose complexities are both $O(m n)$, and thus the main computational complexity of the proposed method is $O\left(\log (m n)+e^{m n}+K m n\right)$, where $K$ is the maximum iteration number. Since LDR and STAR methods requires more loops or iterations, they are time-consuming. From the provided ART and computational complexity analysis, we can conclude that the proposed AGCLDA method guarantees performance while maintaining low complexity.

\section{Conclusion}

In this communication, we propose a joint brightness and sharpness/resolution enhancement method based on the degradation model. First, we build a new degradation model for low-light image, and then introduce the iterative 
back-projection (IBP) mechanism to solve the resulted model efficiently. Besides, a simple and effective pre-processing step is proposed to pre-enhance the low-light input while keeping visual pleasure. In addition, we further accelerate the solution process via logarithmic domain transformation and adjust the brightness via the proposed adaptive gamma correction. In experimental part, the excellent sharpness/resolution, high calculation efficiency, and visual pleasure are verified in terms of various evaluation metrics and in comparison with seven state-of-the-art methods.

\section{Acknowledgement}

This work was supported in part by Key Research and Development Program of Shaanxi (Program No. 2021SF$166)$.

\section{References}

[1] C. Ying, P. Zhao, Y. Li, Low-light-level image super-resolution reconstruction based on iterative projection photon localization algorithm, Journal of Electronic Imaging, 27 (1) (2018) 1-11.

[2] S. M. Pizer, R. E. Johnston, J. P. Ericksen, B. C. Yankaskas, K. E. Muller, Contrast-limited adaptive histogram equalization: speed and effectiveness, in: Proceedings of the First Conference on Visualization in Biomedical Computing, 1990, pp. 337-345.

[3] C. Lee, C. Lee, C. S. Kim, Contrast enhancement based on layered difference representation of 2D histograms, IEEE Transactions on Image Processing, 22 (12) (2013) 5372-5384.

[4] S. Huang, F. Cheng, Y. Chiu, Efficient contrast enhancement using adaptive gamma correction with weighting distribution, IEEE Transactions on Image Processing, 22 (3) (2013) 1032-1041.

[5] X. Fu, D. Zeng, Y. Huang, Y. Liao, X. Ding, J. Paisley, A fusion-based enhancing method for weakly illuminated images, Signal Processing, 129 (2016) 82-96.

[6] Z. Ying, G. Li, Y. Ren, R. Wang, W. Wang, A new low-light image enhancement algorithm using camera response model, in: IEEE International Conference on Computer Vision Workshops (ICCVW), 2017, pp. 3015-3022.

[7] J. Xu, Y. Hou, D. Ren, L. Liu, F. Zhu, M. Yu, H. Wang, L. Shao, STAR: A structure and texture aware Retinex model, IEEE Transactions on Image Processing, 29 (2020) 5022-5037.

[8] Y.-F. Wang, H.-M. Liu, Z.-W. Fu, Low-light image enhancement via the absorption light scattering model, IEEE Transactions on Image Processing, 28 (11) (2019) 5679-5690.

[9] D. Sugimura, T. Mikami, H. Yamashita, T. Hamamoto, Enhancing color images of extremely low light scenes based on RGB/NIR images acquisition with different exposure times, IEEE Transactions on Image Processing, 24 (11) (2015) 3586-3597.

[10] C. Wei, W. Wang, W. Yang, J. Liu, Deep Retinex decomposition for low-light enhancement, in: British Machine Vision Conference, British Machine Vision Association, 2018.

[11] Y. Zhang, J. Zhang, X. Guo, Kindling the darkness: A practical low-light image enhancer, in: Proceedings of the 27th ACM International Conference on Multimedia, MM '19, ACM, 2019, pp. 1632-1640.

[12] J. Wang, W. Tan, X. Niu, B. Yan, RDGAN: Retinex decomposition based adversarial learning for low-light enhancement, in: IEEE International Conference on Multimedia and Expo (ICME), 2019, pp. 1186-1191.

[13] M. V. Afonso, J. M. Bioucas-Dias, M. A. T. Figueiredo, An augmented lagrangian approach to the constrained optimization formulation of imaging inverse problems, IEEE Transactions on Image Processing, 20 (3) (2011) 681-695. 
[14] M. Irani, S. Peleg, Motion analysis for image enhancement: Resolution, occlusion, and transparency, Journal of Visual Communication and Image Representation, 4 (4) (1993) 324-335.

[15] K. He, J. Sun, X. Tang, Guided image filtering, IEEE Transactions on Pattern Analysis and Machine Intelligence 35 (6) (2013) 1397-1409.

[16] M. T. Goodrich, R. Tamassia, M. H. Goldwasser, Data structures and algorithms in Python, John Wiley \& Sons Ltd, 2013.

[17] S. Saitoh, V. K. Tuan, M. Yamamoto, Convolution inequalities and applications, Journal of Inequalities in Pure and Applied Mathematics, 4 (3).

[18] S. Dai, M. Han, Y. Wu, Y. Gong, Bilateral back-projection for single image super resolution, in: IEEE International Conference on Multimedia and Expo, 2007, pp. 1039-1042.

[19] X. Fu, Y. Liao, D. Zeng, Y. Huang, X. Zhang, X. Ding, A probabilistic method for image enhancement with simultaneous illumination and reflectance estimation, IEEE Transactions on Image Processing, 24 (12) (2015) 4965-4977.

[20] X. Fu, D. Zeng, Y. Huang, X. Zhang, X. Ding, A weighted variational model for simultaneous reflectance and illumination estimation, in: IEEE Conference on Computer Vision and Pattern Recognition (CVPR), 2016, pp. 2782-2790.

[21] X. Guo, Y. Li, H. Ling, LIME: Low-light image enhancement via illumination map estimation, IEEE Transactions on Image Processing, 26 (2) (2017) 982-993.

[22] A. Mittal, R. Soundararajan, A. C. Bovik, Making a "completely blind" image quality analyzer, IEEE Signal Processing Letters, $20(3)$.

[23] K. Gu, G. Zhai, W. Lin, X. Yang, W. Zhang, No-reference image sharpness assessment in autoregressive parameter space, IEEE Transactions on Image Processing, 24 (10) (2015) 3218-3231. 